\title{
Fuchsia and Master Integrals for Energy-Energy Correlations at NLO in QCD
}

\section{Oleksandr Gituliar*}

II. Institut für Theoretische Physik, Universität Hamburg, Luruper Chaussee 149, D-22761 Hamburg, Germany

E-mail: oleksandr@gituliar.net

\section{Sven-Olaf Moch}

II. Institut für Theoretische Physik, Universität Hamburg,

Luruper Chaussee 149, D-22761 Hamburg, Germany

E-mail: sven-olaf.moch@desy.de

\begin{abstract}
In this talk we discuss some aspects of the analytical calculation of energy correlations in electronpositron annihilation at a next-to-leading order in QCD. Our primary focus is on the most difficult task: the calculation of master integrals for real-emission contributions, which are functions of two dimensionless variables and the dimensional regulator. We use a method of differential equations and their so-called epsilon-form which is constructed with the help of the FUCHSIA program based on Lee's algorithm.
\end{abstract}

13th International Symposium on Radiative Corrections 24-29 September, 2017

St. Gilgen, Austria

${ }^{*}$ Speaker. 


\section{Introduction}

The energy-energy correlation (EEC) is an observable proposed back in 1978 in [1] to test the consistency of quantum chromodynamics (QCD), when QCD was still considered as "an appealing candidate for the field theory of hadronic interactions". In those times, EEC was considered a very convenient observable to measure from the experimental point of view, since no definition of a jet axis is needed. From the theoretical point of view, EEC is an observable free of mass singularities, as can be easily deduced from its definition,

$$
\Sigma(\xi)=\sum_{a, b} \int \mathrm{dPS} \frac{E_{a} E_{b}}{Q^{2}} \sigma\left(e^{+}+e^{-} \rightarrow a+b+X\right) \delta\left(\xi-\cos \theta_{a b}\right),
$$

since the phase space integration of the cross section $\sigma$ with the measure dPS weighted with the energies $E_{a}$ and $E_{b}$ of partons $a$ and $b$ eliminates all divergences arising from soft and collinear particles production. Nevertheless, the fact that individual diagrams may still diverge requires the use of some regularization scheme, which we choose to be dimensional regularization in $m=4-2 \varepsilon$ space-time dimensions.

Over a time span of almost 40 years EEC was thoroughly studied. Among the recent results is the so far most accurate fixed-order calculation at next-to-next-to-leading order (NNLO) [2] in QCD. This result was subsequently combined with resummation of large logarithms to nextto-next-to-leading logarithmic (NNLL) accuracy and low-energy hadronization effects [3], which allowed for a detailed comparison with available data from LEP.

The focus of our study is twofold. First, we aim to obtain a fully analytical result at next-toleading order (NLO) in QCD which to our surprise is not yet available for the moment. This is in contrast to $N=4$ super-Yang-Mills theory, where analytical expressions for the NLO corrections have recently been derived [4] and one expects that the latter results correspond to those in QCD (after the usual identification of color factors) as far as the polylogarithms of highest weight are concerned. Second, we wish to extend the application of Lee's algorithm [5] and the FUCHSIA program $[6,7]$ beyond univariate problems. To our knowledge, there are no examples of such usage in the literature so far while the method is not limited to univariate problems only. With the NLO integrals for real-emission contributions in EEC at hand, we have an example of a multivariate system of differential equations $[8,9]$, demonstrate its solution with FUCHSIA to obtain the socalled epsilon form [10], and discuss a method to fix the necessary integration constants.

\section{The Method}

The contributions to EEC at NLO in QCD arise from the three electron-positron annihilation sub-processes depicted in Fig. 1. The first ones, the virtual contribution, are rather simple to calculate by directly integrating corresponding matrix elements available from [11]. The last two, on the other hand, form the most difficult part of our calculation. For clarity, we consider here only the first one with gluons, corresponding to the center one Fig. 1:

$$
e^{+}+e^{-} \rightarrow \gamma^{*}(q) \rightarrow q\left(p_{1}\right)+\bar{q}\left(p_{2}\right)+g\left(p_{3}\right)+g\left(p_{4}\right), \quad p_{i}^{2}=0 .
$$

Here, $p_{i}$ denote the light-like final-state particle momenta, while $q$ (with $q^{2}=Q^{2}>0$ ) is the sum of the initial $e^{+}$and $e^{-}$momenta. In addition, we restrict ourselves among all possible combinations 
of correlated particles $a b$, i.e., $q \bar{q}, q g, \bar{q} g$, and $g g$, to the $q g$-correlation only. The corresponding squared matrix elements are generated with FEYNARTS [12] and FORMCALC [13] based on FORM [14]. All the remaining correlations can be calculated in a similar way.

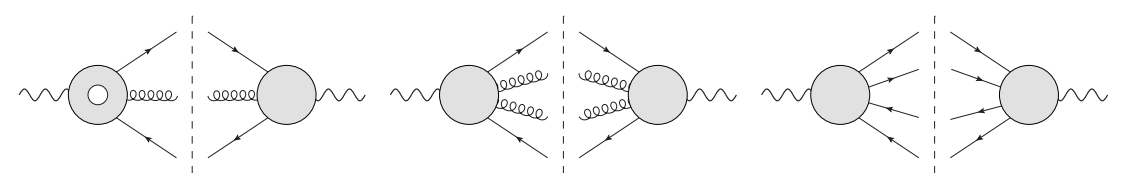

Figure 1: The virtual and the two distinct real emission sub-processes contributing to EEC at NLO.

Let us start with an explicit parametrization of the phase-space in eq. (1.1) in terms of kinematic invariants, which are suitable for a further integral reduction by means of integration-by-parts (IBP) [15]. To that end, we set $q^{2}=1$ and introduce a new, more convenient, angular variable

$$
z=\frac{1-\xi}{2}=\frac{p_{1} \cdot p_{3}}{2 q \cdot p_{1} q \cdot p_{3}}
$$

which, however, still contains non-linear kinematic invariants not yet suitable for an IBP reduction. To fix this, we introduce a new parameter

$$
x=2 q \cdot p_{1}
$$

which leads to the final parametrization of the semi-inclusive three-particle phase-space

$$
\operatorname{dPS}(3 ; x, z)=x q \cdot p_{3} \delta\left(1-x-\left(q-p_{1}\right)^{2}\right) \delta\left(x z q \cdot p_{3}-p_{1} \cdot p_{3}\right) \operatorname{dPS}(3),
$$

where the corresponding inclusive three-particle phase-space $\operatorname{dPS}(3)$ is given by

$$
\operatorname{dPS}(3)=\mathrm{d}^{m} p_{1} \delta\left(p_{1}^{2}\right) \mathrm{d}^{m} p_{2} \delta\left(p_{2}^{2}\right) \mathrm{d}^{m} p^{3} \delta\left(p_{3}^{2}\right) \delta\left(\left(q-p_{1}-p_{2}-p_{3}\right)^{2}\right) .
$$

With this parametrization IBP rules for the corresponding real-emission phase space integrals can be applied to derive differential equations for the set of master integrals. These master integrals now depend on the two scale-less parameters $x$ and $z$, which is an added complication. However, we will see later this can be easily resolved.

\subsection{IBP Reduction for $F(x, z, \varepsilon)$}

In the IBP reduction of real-emission phase space integrals all delta functions in eq. (2.5) are treated as cut propagators according to the Cutkosky rules. We use the program LiTERED [16, 17] for this task, which produces a family of 11 master integrals $F_{i}(x, z, \varepsilon)$ defined as

$$
\begin{array}{llll}
F_{1}=\{\} & F_{2}=\{2\} & F_{3}=\{2,2\} & F_{4}=\{2,6\} \\
F_{5}=\{1,2\} & F_{6}=\{5\} & F_{7}=\{1,4,5\} & F_{8}=\{2,3,4\}, \\
F_{9}=\{2,5\} & F_{10}=\{3,5\} & F_{11}=\{2,4,5\} &
\end{array}
$$

where phase space integration according to eq. (2.4) is understood. The integrals $F_{2}, F_{3}$, and $F_{4}$ form a coupled sub-system and the denominators are defined ${ }^{1}$ as

$$
\begin{aligned}
& D_{1}=\left(p_{2}+p_{3}\right)^{2} \quad D_{2}=\left(q-p_{2}\right)^{2} \quad D_{3}=\left(q-p_{1}-p_{2}\right)^{2} \\
& D_{4}=\left(q-p_{1}-p_{3}\right)^{2} \quad D_{5}=\left(q-p_{2}-p_{3}\right)^{2} \quad D_{6}=p_{1}^{2} \quad D_{7}=q \cdot p_{3} \cdot
\end{aligned}
$$

\footnotetext{
${ }^{1}$ Note, that $D_{6}$ corresponds to the on-shell delta function $\delta\left(p_{1}^{2}\right)$ and denotes its additional power when used in the parametrization of $F_{4}$, e.g, $\delta^{2}\left(p_{1}^{2}\right)$.
} 


\subsection{Differential Equations for $F(x, z, \varepsilon)$}

With the complete set of IBP reduction rules at hand we can easily construct a system of differential equations. In our approach we consider ordinary differential equations, where only one variable is free and the rest are treated as symbolic constants. At this stage we choose $z$ as a free variable, which leads to a system of equations

$$
\frac{\mathrm{d} F(x, z, \varepsilon)}{\mathrm{d} z}=A(x, z, \varepsilon) F(x, z, \varepsilon)
$$

with singular points (alphabet) in

$$
0, \quad 1, \quad \frac{1}{x}, \frac{1}{x(x-2)}
$$

To solve this system we find its epsilon form [10] with FUCHSIA [6, 7] (see also [18, 19, 20]) and then integrate it to any order in $\varepsilon$ using a recursive definition of hyperlogarithms (see [21] and references therein). The resulting new system takes the form

$$
\frac{\mathrm{d} \hat{F}(x, z, \varepsilon)}{\mathrm{d} z}=\varepsilon B(x, z) \hat{F}(x, z, \varepsilon),
$$

with the relation between new and old bases given as

$$
F(x, z, \varepsilon)=T(x, z, \varepsilon) \hat{F}(x, z, \varepsilon)
$$

where the matrix $T(x, z, \varepsilon)$ has been found automatically by FUCHSIA.

For clarity, let us provide here the solution to one of master integrals in eq. (2.6),

$$
\begin{aligned}
& F_{4}(x, z, \varepsilon)=\frac{1}{15 \varepsilon^{2}}\left(C_{3}^{0}(x)-2 C_{2}^{0}(x)\right)+\frac{1}{30 \varepsilon}\left(\left(15 C_{1}^{0}(x)+4 C_{2}^{0}(x)\right.\right. \\
& \left.-6 x C_{3}^{0}(x)-2 x C_{4}^{0}(x)\right) \mathrm{H}_{0}(z)+\left(\frac{15}{1-x} C_{1}^{0}(x)+2 C_{3}^{0}(x)\right. \\
& \left.-2 x C_{4}^{0}(x)\right) \mathrm{H}_{1}(z)+\left(\frac{15(x-2)}{1-x} C_{1}^{0}(x)+20 C_{2}^{0}(x)+4 x C_{4}^{0}(x)\right. \\
& \left.+2(3 x-7) C_{3}^{0}(x)\right) \mathrm{H}_{1 / x}(z)+\frac{2\left(13 x z^{2}-17 x z+3 x+z\right)}{x z(1-z)} C_{3}^{0}(x) \\
& -\frac{15(1-2 z)}{x z(1-z)} C_{1}^{0}(x)+\frac{4(13 x z-1)}{x z} C_{2}^{0}(x)+\frac{2(x z-2 z+1)}{z(1-z)} C_{4}^{0}(x) \\
& \left.-4 C_{2}^{1}(x)+2 C_{3}^{1}(x)\right)+\mathscr{O}\left(\varepsilon^{0}\right),
\end{aligned}
$$

where the polylogarithms $\mathrm{H}_{\vec{w}}$ are recursively defined as

$$
\mathrm{H}_{a, \vec{w}}(z)=\int_{0}^{z} \frac{\mathrm{d} z^{\prime}}{z^{\prime}-a} \mathrm{H}_{\vec{w}}\left(z^{\prime}\right)
$$




\subsection{Integration Constants for $F(x, z, \varepsilon)$}

The unknown integration constants $C(x)$ in eq.(2.12) are functions of $x$ and still need to be found. For this purpose we employ the following relation

$$
F_{i}^{\star}(x, \varepsilon)=\int_{0}^{1} \mathrm{~d} z f_{i}(z) F_{i}(x, z, \varepsilon) .
$$

Here, the idea is the following: If we know both sides of this relation we can derive a system of linear equations with $C(x)$ as unknown functions by requiring that coefficients in front of identical polylogarithms on both sides of eq. (2.14) are equal. Solutions of this system provide those unknown integration constants $C(x)$.

The integration on the right-hand side of eq. (2.14) can be done with the help of the HYPERINT package [22]. However, particular attention should be paid at this stage since some of these integrals diverge (like in the case of $F_{4}(x, z, \varepsilon)$ above). We account for this by introducing additional $z$-dependent pre-factors $f(z)$ for each of the master integrals in eq. (2.6). These are listed in the table:

\begin{tabular}{|c|c|c|c|c|c|c|c|c|c|c|c|}
\hline$i$ & 1 & 2 & 3 & 4 & 5 & 6 & 7 & 8 & 9 & 10 & 11 \\
\hline$f_{i}$ & 1 & 1 & $z$ & $z(1-z)$ & 1 & $z^{2}$ & $1-z$ & $z$ & $z$ & $z$ & $z(1-z)$ \\
\hline
\end{tabular}

In this way, all the integrals on the right-hand side of eq. (2.14) become finite and can be integrated.

\subsection{IBP Reduction for $G(x, \varepsilon)$}

For the left-hand side of eq. (2.14) we can introduce a new IBP basis, since the integration over $z$ eliminates one delta function in eq. (2.4) and leads to a new three-particle phase-space given by

$$
\mathrm{dPS}(3 ; x)=\int_{0}^{1} \mathrm{~d} z \mathrm{dPS}(3 ; x, z)=\mathrm{dPS}(3) \delta\left(1-x-\left(q-p_{1}\right)^{2}\right) .
$$

We find the IBP reduction rules for this basis, again using LITERED, which leads to 12 master integrals defined as

$$
\begin{aligned}
& G_{1}=\{\} \quad G_{2}=\{2\} \quad G_{3}=\{7\} \quad G_{4}=\{2,7\} \\
& G_{5}=\{2, \varnothing, 7\} \quad G_{6}=\{1,2\} \quad G_{7}=\{2,3,4,7\} \quad G_{8}=\{5,7\} \quad \text {. } \\
& G_{9}=\{2,4,5\} \quad G_{10}=\{2,4,5,7\} \quad G_{11}=\{3,5,7\} \quad G_{12}=\{1,4,5,7\}
\end{aligned}
$$

where phase space integration according to eq. (2.15) is again implied. In this way we can express the left-hand side of eq. (2.14) by using only these masters. For example,

$$
\begin{aligned}
& F_{4}^{\star}(x, \varepsilon)=\frac{(2-3 \varepsilon)\left(x+5 \varepsilon x-2 \varepsilon^{2}(8-7 x)\right)}{4 \varepsilon^{2} x^{2}(4-5 x)} G_{1}(x, \varepsilon) \\
& \quad+\frac{x(1-x)+\varepsilon\left(16-33 x+15 x^{2}\right)-\varepsilon^{2}\left(48-82 x+26 x^{2}\right)}{4 \varepsilon x^{2}(4-5 x)} G_{2}(x, \varepsilon) \\
& \quad+\frac{(1-2 \varepsilon)(x-2 \varepsilon(2-x))}{4 \varepsilon x(4-5 x)} G_{3}(x, \varepsilon) \\
& \quad-\frac{4-7 x+2 x^{2}+\varepsilon x(4-2 x)}{4 x(4-5 x)} G_{4}(x, \varepsilon)-\frac{3 x(1-x)}{4(4-5 x)} G_{5}(x, \varepsilon) .
\end{aligned}
$$




\subsection{Differential Equations for $G(x, \varepsilon)$}

Next, we compute the master integrals in eq. (2.16) by solving the corresponding system of differential equations, but this time in $x$ variable. As before, we find its epsilon form using FUCHSIA and solve the resulting system to desired order in $\varepsilon$ by applying the recursive definition of polylogarithms in eq. (2.13). For example, the solution to $G_{5}$ looks as follows:

$$
\begin{aligned}
& G_{5}(x, \varepsilon)=\frac{2}{x \varepsilon^{2}}\left(30 C_{1}^{0}+6 C_{2}^{0}+6 C_{3}^{0}+(14-35 x) C_{4}^{0}-2 C_{5}^{0}\right)+\frac{1}{x \varepsilon}\left(+60 C_{1}^{1}\right. \\
& -390 C_{1}^{0}-78 C_{2}^{0}+12 C_{2}^{1}-78 C_{3}^{0}+12 C_{3}^{1}-(182-455 x) C_{4}^{0}+(28-70 x) C_{4}^{1} \\
& +26 C_{5}^{0}-4 C_{5}^{1}+\left((60-120 x) C_{1}^{0}+(132-144 x) C_{2}^{0}-(48-36 x) C_{3}^{0}\right. \\
& \left.-(112-84 x) C_{4}^{0}+(16-12 x) C_{5}^{0}\right) \mathrm{H}_{0}(x)+\left((-480+120 x) C_{1}^{0}-(96-144 x) C_{2}^{0}\right. \\
& \left.\left.-36(1+x) C_{3}^{0}+(-224+336 x) C_{4}^{0}+(-8+12 x) C_{5}^{0}\right) \mathrm{H}_{1}(x)\right)+\mathscr{O}\left(\varepsilon^{0}\right) .
\end{aligned}
$$

In this case, the integration constants $C_{i}^{0,1}$ are now just numbers.

\subsection{Integration Constants for $G(x, \varepsilon)$}

In order to obtain the integration constants for the integrals $G(x, \varepsilon)$ we use the same technique as for $F(x, z, \varepsilon)$ before. We define a relation

$$
G_{i}^{\star}(\varepsilon)=\int_{0}^{1} \mathrm{~d} x g_{i}(x) G_{i}(x, \varepsilon)
$$

and integrate its right-hand side with HYPERINT using now $x$-dependent pre-factors $f(x)$ to regularize the denominators in $x$. For each of the master integrals in eq. (2.16) they are given in the table:

\begin{tabular}{|c|c|c|c|c|c|c|c|c|c|c|c|c|}
\hline$i$ & 1 & 2 & 3 & 4 & 5 & 6 & 7 & 8 & 9 & 10 & 11 & 12 \\
\hline$g_{i}$ & 1 & 1 & 1 & 1 & $x$ & 1 & $(1-x)^{2}$ & 1 & $x$ & $x(1-x)$ & $1-x$ & $1-x$ \\
\hline
\end{tabular}

\subsection{IBP Reduction for $H(\varepsilon)$}

For the last iteration, that is the computation of the left-hand side of eq. (2.19) we introduce a new IBP basis, since, as before, the integration eliminates one delta function and the new phasespace becomes

$$
\int_{0}^{1} \mathrm{~d} x \mathrm{dPS}(3 ; x)=\mathrm{dPS}(3)
$$

cf. eq. (2.5). We find the necessary IBP reduction rules for this basis again with LITERED and only two master integrals remain,

$$
H_{1}=\{\} \quad H_{2}=\{1,2,7\} .
$$

These are sufficient to determine the left-hand side of eq. (2.19). For example, we find

$$
G_{5}^{\star}(\varepsilon)=-\frac{2(2-3 \varepsilon)(3-4 \varepsilon)\left(1-7 \varepsilon+30 \varepsilon^{2}-36 \varepsilon^{3}\right)}{3 \varepsilon^{2}\left(1-5 \varepsilon+6 \varepsilon^{2}\right)} H_{1}(\varepsilon) .
$$




\section{Results}

We can now summarize all the results for the master integrals $H(\varepsilon), G(x, \varepsilon)$ and $F(x, z, \varepsilon)$ in this order by subsequently substituting the solutions for $H(\varepsilon)$ back into $G(x, \varepsilon)$ and $G(x, \varepsilon)$ back into $F(x, z, \varepsilon)$.

\subsection{Master Integrals $H(\varepsilon)$}

The integrals in eq. (2.21) coincide with fully inclusive phases-space integrals. The first one is known in the literature [23], i.e.,

$$
\begin{aligned}
H_{1}(\varepsilon)=\frac{1}{12}+\frac{59}{72} \varepsilon+\left(\frac{2263}{432}\right. & \left.-\frac{2}{3} \zeta_{2}\right) \varepsilon^{2}+\left(\frac{72023}{2592}-\frac{59}{9} \zeta_{2}-\frac{13}{6} \zeta_{3}\right) \varepsilon^{3} \\
& +\left(\frac{2073631}{15552}-\frac{2263}{54} \zeta_{2}-\frac{767}{36} \zeta_{3}+\frac{1}{12} \zeta_{4}\right) \varepsilon^{4}+\mathscr{O}\left(\varepsilon^{5}\right)
\end{aligned}
$$

and we calculate the second one explicitly as:

$$
H_{2}(\varepsilon)=-\frac{4 \zeta_{3}}{\varepsilon}-42 \zeta_{4}+\mathscr{O}(\varepsilon)
$$

\subsection{Master Integrals $G(x, \varepsilon)$}

Next, we substitute eqs. (3.1)-(3.2) into eq. (2.22) which delivers us the left-hand side of eq. (2.19). The right-hand side was integrated with HYPERINT and we can determine the integration constants in eq. (2.18). The result is given by

$$
\begin{aligned}
& G_{5}(x, \varepsilon)=\frac{1}{3 x}\left[-\frac{1}{\varepsilon^{2}}+\frac{\mathrm{H}_{0}(x)+4 \mathrm{H}_{1}(x)}{\varepsilon}-(7-6 x) \mathrm{H}_{0,0}(x)-2(5-3 x) \mathrm{H}_{0,1}(x)\right. \\
& \quad-2(2+3 x) \mathrm{H}_{1,0}(x)-2(5+3 x) \mathrm{H}_{1,1}(x)-2(1-3 x) \zeta_{2}+\left((61-54 x) \mathrm{H}_{0,0,0}(x)\right. \\
& \quad+(46-36 x) \mathrm{H}_{0,0,1}(x)+4 \mathrm{H}_{0,1,0}(x)+28 \mathrm{H}_{0,1,1}(x)-18 x \mathrm{H}_{0,1,1}(x)+4 \mathrm{H}_{1,0,0}(x) \\
& \quad+18 x \mathrm{H}_{1,0,0}(x)+16 \mathrm{H}_{1,0,1}(x)+4 \mathrm{H}_{1,1,0}(x)+36 x \mathrm{H}_{1,1,0}(x)+10 \mathrm{H}_{1,1,1}(x) \\
& \left.\left.\quad+54 x \mathrm{H}_{1,1,1}(x)+\zeta_{2}\left(38 \mathrm{H}_{0}(x)-36 x \mathrm{H}_{0}(x)-16 \mathrm{H}_{1}(x)\right)+(36-18 x) \zeta_{3}\right) \varepsilon\right]+\mathscr{O}\left(\varepsilon^{2}\right) .
\end{aligned}
$$

In this way we can obtain the entire set of integrals in eq. (2.16).

\subsection{Master Integrals $F(x, z, \varepsilon)$}

Finally, we can compute the left-hand side of eq. (2.14) by using IBP reduction rules similar to eq. (2.17) and find the remaining unknown integration constants $C(x, \varepsilon)$ in eq. (2.12). As an 
example, the result for $F_{11}$ reads

$$
\begin{aligned}
& F_{11}(x, z, \varepsilon)=\frac{4}{3(1-x) x^{2}(1-z) z}\left[\frac{3+x-4 x z}{2 \varepsilon^{2}}+\frac{1}{\varepsilon}\left(-2(3+x-4 x z) \mathrm{H}_{1}(x)\right.\right. \\
& \quad-(6-x-5 x z) \mathrm{H}_{0}(x)-(3+x-4 x z) \mathrm{H}_{0}(z)-(3-x-2 x z) \mathrm{H}_{1}(z) \\
& \left.\quad+6(1-x z) \mathrm{H}_{1 / x}(z)\right)+9(1-x) \mathrm{H}_{0,1}(x)+8(3+x-4 x z) \mathrm{H}_{1,1}(x)+(24 \\
& \quad-7 x-17 x z) \mathrm{H}_{0,0}(x)+\left(2(6-x-5 x z) \mathrm{H}_{0}(z)+4(3-x-2 x z) \mathrm{H}_{1}(z)\right. \\
& \left.-15(1-x z) \mathrm{H}_{\frac{1}{x}}(z)-3(1-x z) \mathrm{H}_{\frac{1}{x(2-x)}}(z)\right) \mathrm{H}_{0}(x)+\mathrm{H}_{0}(1-x)(5(3+x \\
& -4 x z) \mathrm{H}_{0}(x)+4(3+x-4 x z) \mathrm{H}_{0}(z)+4(3-x-2 x z) \mathrm{H}_{1}(z)-21(1 \\
& \left.-x z) \mathrm{H}_{\frac{1}{x}}(z)-3(1-x z) \mathrm{H}_{\frac{1}{x(2-x)}}(z)\right)+2(3+x-4 x z) \mathrm{H}_{0,0}(z)+2(3-x \\
& -2 x z) \mathrm{H}_{0,1}(z)-12(1-x z) \mathrm{H}_{0, \frac{1}{x}}(z)+2(3-x-2 x z) \mathrm{H}_{1,0}(z)+2(3-2 x \\
& -x z) \mathrm{H}_{1,1}(z)-6(2-x-x z) \mathrm{H}_{1, \frac{1}{x}}(z)-9(1-x z) \mathrm{H}_{\frac{1}{x}, 0}(z)-6(1-x z) \\
& \quad \times \mathrm{H}_{\frac{1}{x}, 1}(z)+15(1-x z) \mathrm{H}_{\frac{1}{x}, \frac{1}{x}}(z)-3(1-x z) \mathrm{H}_{\frac{1}{x(2-x)}, 0}(z)+3(1-x z) \\
& \left.\quad \times \mathrm{H}_{\frac{1}{x(2-x)}, \frac{1}{x}}(z)+2(3-5 x+2 x z) \zeta_{2}\right]+\mathscr{O}(\varepsilon) .
\end{aligned}
$$

This is the final result computed to sufficient depth in $\varepsilon$ which can then be further used to calculate the EEC defined in eq. (1.1).

\section{Summary}

In this talk we have presented the NLO real-emission master integrals for the quark-gluon EEC in electron-positron annihilation in QCD. They are expressed in terms of polylogarithms of two variables. Real-emission integrals are the most complicated pieces on the way to obtain the complete result for EEC at NLO, hence their calculation is a crucial task. To that end we have derived the epsilon form for the corresponding systems of differential equation in a fully automatic manner using the implementation in the FUCHSIA program, which is based on Lee's algorithm. We have shown that FUCHSIA and the method are, in general, indeed suitable to solve differential equations with multiple dimensionless variables, a fact that has not been mentioned in the literature so far.

\section{Acknowledgment}

We are thankful to Vitaly Magerya for carefully reading this paper and useful comments. The Feynman diagrams have been drawn with Axodraw [24].

This work has been supported by the Deutsche Forschungsgemeinschaft in Sonderforschungsbereich 676 Particles, Strings, and the Early Universe. 


\section{References}

[1] C. L. Basham, L. S. Brown, S. D. Ellis, and S. T. Love. Energy Correlations in Electron-Positron Annihilation in Quantum Chromodynamics: Asymptotically Free Perturbation Theory. Phys. Rev., D19:2018, 1979.

[2] V. Del Duca, C. Duhr, A. Kardos, G. Somogyi, and Z. Trocsanyi. Three-Jet Production in Electron-Positron Collisions at Next-to-Next-to-Leading Order Accuracy. Phys. Rev. Lett., 117(15):152004, 2016.

[3] Z. Tulipant, A. Kardos, and G. Somogyi. Energy-energy correlation in electron-positron annihilation at NNLL+NNLO accuracy. arXiv:1708.04093.

[4] A. V. Belitsky, S. Hohenegger, G. P. Korchemsky, E. Sokatchev, and A. Zhiboedov. Energy-Energy Correlations in N=4 Supersymmetric Yang-Mills Theory. Phys. Rev. Lett., 112(7):071601, 2014.

[5] R. Lee. Reducing differential equations for multiloop master integrals. JHEP, 04:108, 2015.

[6] O. Gituliar and V. Magerya. Fuchsia and master integrals for splitting functions from differential equations in QCD. PoS, LL2016:030, 2016.

[7] O. Gituliar and V. Magerya. Fuchsia: a tool for reducing differential equations for Feynman master integrals to epsilon form. Comput. Phys. Commun., 219:329-338, 2017.

[8] A. V. Kotikov, Differential equations method: The Calculation of vertex type Feynman diagrams, Phys. Lett. B259:314-322, 1991.

[9] A. V. Kotikov, Differential equations method: New technique for massive Feynman diagrams calculation, Phys. Lett. B254:158-164, 1991.

[10] J. Henn. Multiloop integrals in dimensional regularization made simple. Phys. Rev. Lett., 110:251601, 2013.

[11] L. W. Garland, T. Gehrmann, E. W. N. Glover, A. Koukoutsakis, and E. Remiddi. The Two loop QCD matrix element for $e^{+} e^{-} \rightarrow 3$ jets. Nucl. Phys., B627:107-188, 2002.

[12] T. Hahn. Generating Feynman diagrams and amplitudes with FeynArts 3. Comput. Phys. Commun., 140:418, 2001

[13] T. Hahn and M. Perez-Victoria. Automatized one loop calculations in four-dimensions and D-dimensions. Comput. Phys. Commun., 118:153, 1999.

[14] J. Vermaseren. New features of FORM. math-ph/0010025.

[15] K. G. Chetyrkin and F. V. Tkachov, Integration by Parts: The Algorithm to Calculate beta Functions in 4 Loops. Nucl. Phys., B192:159, 1981.

[16] R. Lee. Presenting LiteRed: a tool for the Loop InTEgrals REDuction. arXiv:1212.2685.

[17] R. Lee. LiteRed 1.4: a powerful tool for reduction of multiloop integrals. J.Phys.Conf.Ser., 523:012059, 2014.

[18] M. Prausa. epsilon: A tool to find a canonical basis of master integrals. Comput. Phys. Commun., 219:361, 2017.

[19] C. Meyer. Transforming differential equations of multi-loop Feynman integrals into canonical form. JHEP 1704:006, 2017.

[20] C. Meyer. Algorithmic transformation of multi-loop master integrals to a canonical basis with CANONICA. arXiv:1705.06252 [hep-ph]. 
[21] E. Panzer. Feynman integrals and hyperlogarithms. PhD thesis, Humboldt U., Berlin, Inst. Math., 2015.

[22] E. Panzer. Algorithms for the symbolic integration of hyperlogarithms with applications to Feynman integrals. Comput. Phys. Commun., 188:148-166, 2015.

[23] A. Gehrmann-De Ridder, T. Gehrmann, and G. Heinrich. Four particle phase space integrals in massless QCD. Nucl. Phys., B682:265-288, 2004.

[24] J. Vermaseren. Axodraw. Comput. Phys. Commun. 83:45-58, 1994. 